\title{
Gout Masquerading as a Triquetral Fracture
}

NICHOLAS KORTAN, DO; JEFFERSON ROBERTS, MD; JONATHAN ROEBUCK, MD, Department of Rheumatology, Walter Reed Army Medical Center, Bldg 2, 6900 Georgia Ave. NW, Washington, DC 20307, USA. Address correspondence to Dr. Kortan; E-mail: Nicholas.kortan@amedd.army.mil. J Rheumatol 2010;37:670-1; doi:10.3899/jrheum.091000

The differential for unilateral hand swelling in the adult includes crystalline arthropathy, septic arthritis, musculoskeletal injury, and other inflammatory conditions. Tools used to establish a diagnosis include arthrocentesis for synovial fluid analysis as well as radiographic imaging. We describe a case of gouty arthritis of the hand masquerading as a triquetral fracture on plain radiograph.

A 61-year-old man initially presented to orthopedics with nontraumatic acute unilateral hand swelling (Figure 1) and was diagnosed with a triquetral fracture on plain radiograph (Figure 2). Despite immobilization and conservative therapy, he had persistent swelling and was referred to rheumatology. We were suspicious for crystalline arthropathy, given an elevated uric acid level and nonfocal swelling. Needle aspiration of the wrist demonstrated numerous intracellular negatively birefringent crystals under polarized light microscopy. An intraarticular corticosteroid injection to treat gouty arthritis was considered; however, we were concerned about impeding wound-healing in the setting of a persistent fracture. This prompted additional imaging with computed tomography $(\mathrm{CT})^{2}$, which to our surprise did not show a triquetral fracture but only numerous nonspecific radio-opaque deposits along the inferior border of the triquetral bone, with associated generalized soft-tissue swelling (Figure 3). An intraarticular corticosteroid injection was then administered, which provided a prompt clinical response. A followup CT after 6 months of urate-lowering therapy demonstrated radiological resolution (Figure 4).

We are not aware of any previously reported cases of gout hidden as a fracture on plain radiograph. Our case demonstrates how gout can masquerade as an apparent fracture or "pseudo-fracture." Certainly, joint aspiration and synovial fluid analysis should be performed in an acute monoarticular and/or oligoarticular arthritis, but most often in the cases of nontraumatic joint pain and swelling ${ }^{1}$. Intraarticular corticosteroid injected into sites of osteochondral fractures is contraindicated ${ }^{1,3}$. In our case, CT was invaluable in differentiating between fracture and a crystalline arthropathy, namely gout.

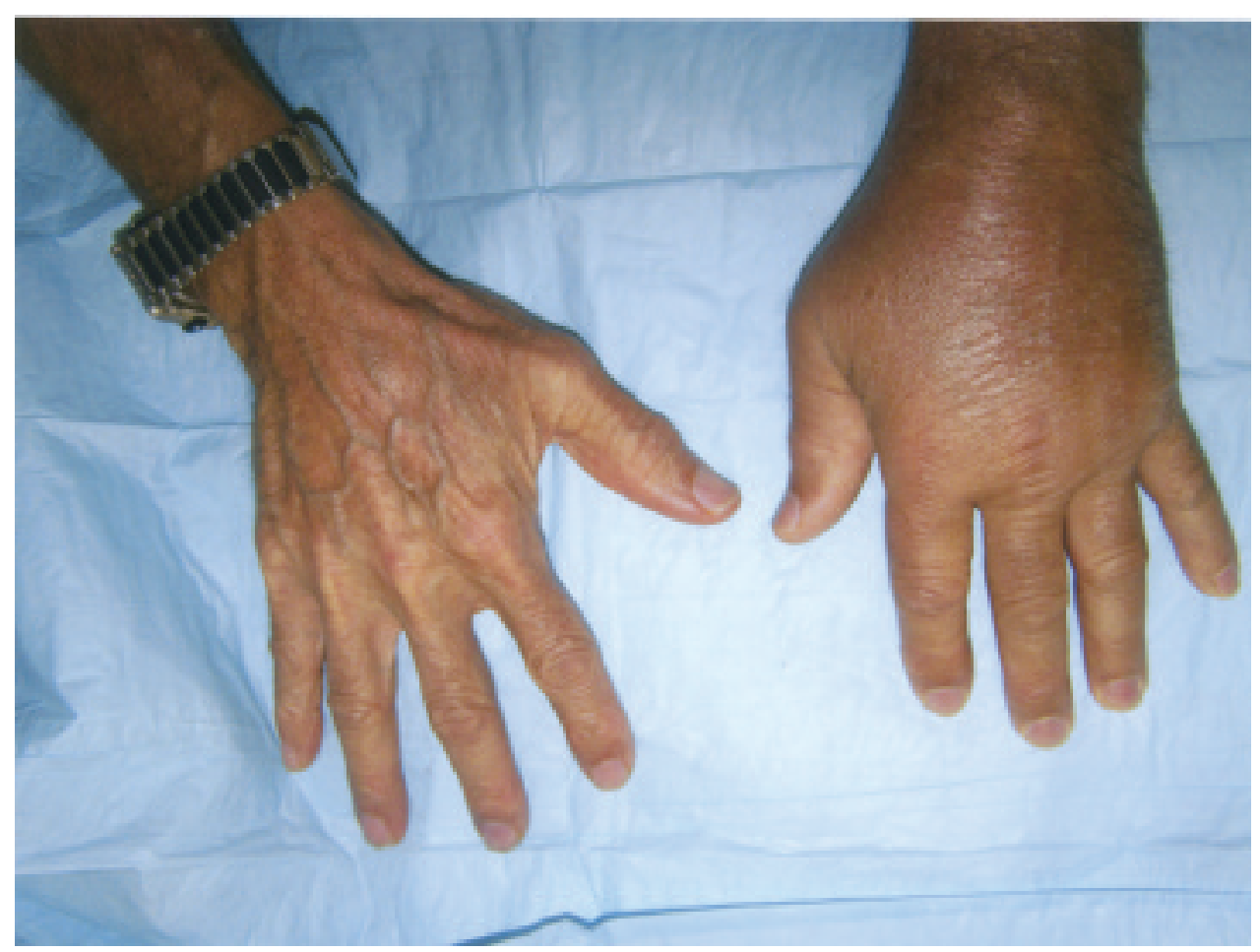

Figure 1. Unilateral swelling of left hand. 


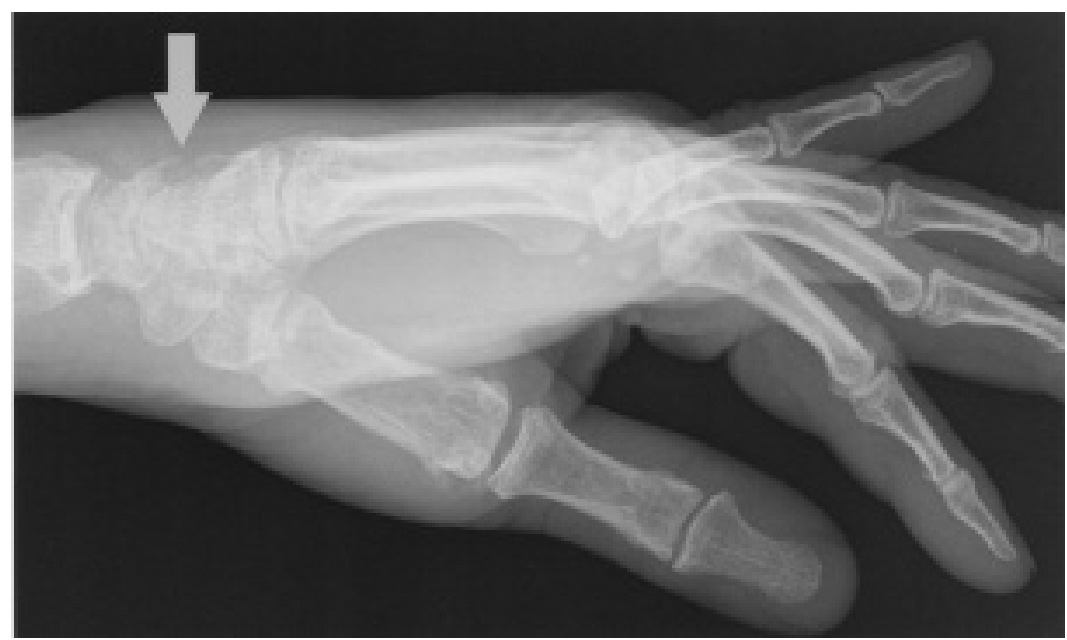

Figure 2. Plain radiograph of left hand, lateral view, showing cortical irregularity (arrow) with overlying soft tissue swelling, suspicious for triquetral fracture.

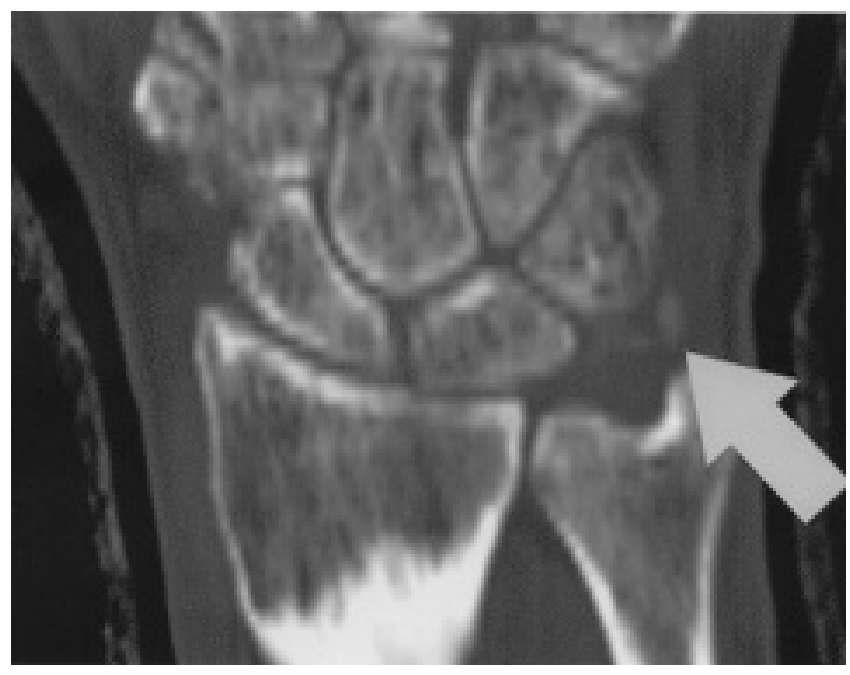

Figure 3. Noncontrast CT scan of left hand, coronal section, showing amorphous radio-opaque deposits (arrow) along the inferior border of the triquetral bone. No fracture is identified.

\section{REFERENCES}

1. Roberts JR, Hedges JR. Arthrocentesis. In: Roberts JR, Hedges JR. Clinical procedures in emergency medicine. 4th ed. Philadelphia: Saunders; 2004.

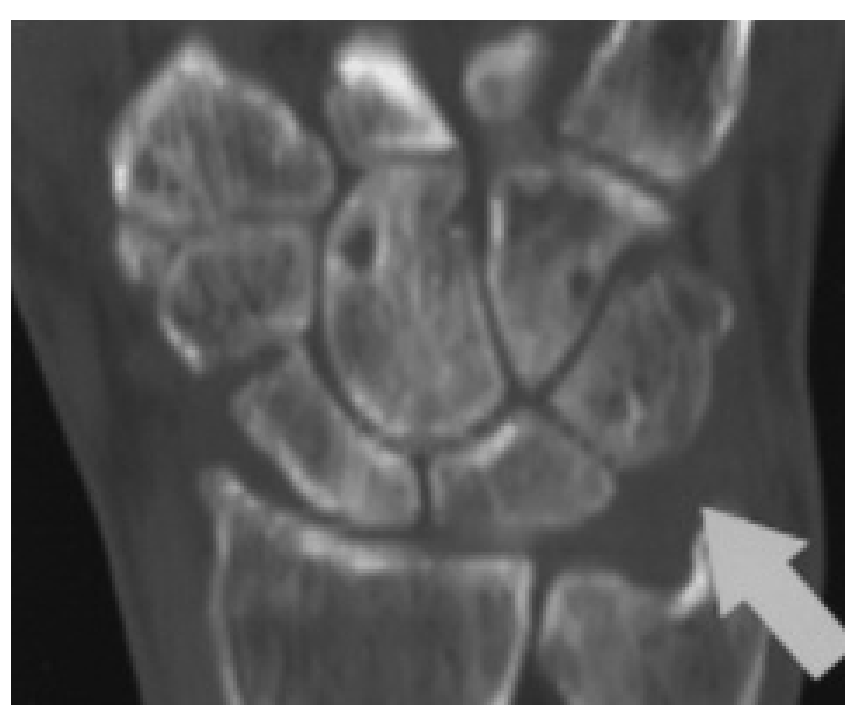

Figure 4. Followup noncontrast CT scan of left hand, coronal section, showing resolution of the previously seen amorphous radio-opaque deposits.

2. Monu JU, Pope TL Jr. Gout: A clinical and radiological review. Radiol Clin North Am 2004;42:169-84.

3. DeLee JC, Drez D Jr, Miller MD. Wrist and hand. In: DeLee and Drez's orthopaedic sports medicine. 3rd ed. St. Louis: Saunders; 2009. 